\title{
Otimização de Semáforos para Tráfego Terrestre com Estratégias Evolutivas
}

\author{
Lucas de Carvalho Gomes ${ }^{1}$, Luís Henrique M. K. Costa ${ }^{1}$ \\ ${ }^{1}$ Grupo de Teleinformática e Automação (GTA - PEE/COPPE/UFRJ) \\ \{gomes, luish\}@gta.ufrj.br
}

\begin{abstract}
Optimizing traffic lights for land vehicle traffic is a task of great relevance, since the occupation of urban pathways is currently increasing in urban environments, consequently increasing delays, traffic jams and their consequential losses. Reacting to this issue, a significant part of the related work considers vehicular traffic to the detriment of pedestrians. However, the higher their waiting time, the more pedestrians adopt risky behavior. Aiming to treat the issue considering the conflicting interests of vehicles and pedestrians, this work performs the optimization of traffic lights considering the average delays of both types of users, through an Evolution Strategy. The problem is modeled on the basis of reference works of the area. The approach has found solutions that keep the pedestrian delays within the limits given by related work.
\end{abstract}

Resumo. A otimização de semáforos para tráfego terrestre é uma tarefa de grande relevância, dado que a ocupação das vias urbanas cresce nos centros urbanos, aumentando os atrasos e congestionamentos, e seus consequentes prejuízos. Em reação, trabalhos relacionados considera o tráfego veicular em detrimento dos pedestres. Contudo, quanto maior o tempo de espera destes, mais medidas arriscadas eles tomam, aumentando a incidência de acidentes. Visando tratar o problema considerando os interesses conflitantes dos veículos e pedestres, este trabalho realiza a otimização de semáforos considerando os atrasos médios de ambos, através de uma Estratégia Evolutiva. O problema é modelado a partir de trabalhos de referência na área. A abordagem conseguiu encontrar soluções que mantêm o atraso dos pedestres dentro dos limites dados pela literatura.

\section{Introdução}

O aumento do número de veículos em circulação sobrecarrega as vias urbanas, gerando não apenas engarrafamentos mas prejuízos econômicos, ambientais e à saúde dos habitantes. Uma evidência quantitativa é o estudo realizado pela empresa INRIX Research com dados fornecidos por mais de 200 cidades, que indica perdas severas de capital e tempo por congestionamentos [Reed and Kidd 2019]. Nos Estados Unidos, o setor de transporte de carga perde US\$74,5 bilhões por ano. Têm impacto nisto os semáforos urbanos, que regulam a passagem de veículos e pedestres. Logo, a sua otimização atrai a atenção da academia, da indústria e de governos, que há décadas buscam soluções eficientes [Yang and Benekohal 2011, Schmöcker et al. 2008, Ma et al. 2015, Sanchez-Medina et al. 2010] A maior parte das técnicas otimiza métricas como o tempo médio de viagem, o atraso em relação ao tempo de viagem ideal ou o consumo de combustível [Jin et al. 2017]. No 
entanto, raramente se considera a satisfação dos pedestres, o que é uma limitação: quanto maior o tempo de espera, mais impaciente fica o pedestre e mais risco ele corre para a travessia, aumentando os acidentes [Vallyon et al. 2008, Virkler 1998, Brilon 1994]. Considerá-los na otimização também permite um ajuste mais fino dos semáforos, o que pode beneficiar todos os usuários.

Trabalhos relacionados implementam a otimização por modelos analíticos e simulações, que se subdividem em abordagens macroscópicas - aproximam a dinâmica do tráfego urbano por meio de conceitos da Dinâmica dos Fluidos -; microscópicas - representam o comportamento do tráfego através de suas entidades individuais, veículos e pedestres, com modelos teóricos de mobilidade -; e mesoscópicas, intermediárias entre as duas anteriores. Abordagens macroscópicas são geralmente mais rápidas porém menos acuradas. Por outro lado, as microscópicas têm maior fidelidade de representação mas requerem mais recursos computacionais, pois são simulações. Portanto, uma dificuldade das soluções com simulações microscópicas é restringir o tempo de otimização para que elas sejam compatíveis com a operação em tempo real.

O problema aqui considerado é a otimização das temporizações de semáforos em interseções para agilizar a passagem de veículos e pedestres. Há duas maneiras de manejar os pedestres em um cruzamento [Yang 2010]. A abordagem Two-Way Crossing (TWC) permite a passagem simultânea de pedestres e veículos cujas trajetórias não conflitam. Já a Exclusive Pedestrian Phase (EPP), ou Fase Exclusiva de Pedestres, mantém veículos e pedestres em fases separadas, reduzindo a chance de acidentes, mas tendendo a aumentar os atrasos de veículos e pedestres [Ishaque and Noland 2005].

Neste contexto, este trabalho implementa a otimização da temporização de semáforos, considerando os interesses conflitantes de pedestres e veículos, a partir de simulações de tráfego microscópicas executadas na ferramenta SUMO (Simulation of Urban Mobility) [Lopez et al. 2018]. Foram implementados dois cenários de simulação, de tamanhos distintos. Um usa a abordagem TWC; o outro, a EPP. Uma modelagem para o problema foi desenvolvida neste trabalho de acordo com dados de publicações relacionadas; a partir dela, o problema foi resolvido a partir de uma Estratégia Evolutiva (EE) [Beyer and Schwefel 2002]. Com esta configuração, cada avaliação da função objetivo corresponde a uma simulação, causando um grande custo computacional. Por isto, a EE utilizada aqui recebeu diversos ajustes, de modo a reduzir a quantidade de avaliações da função objetivo (FES - Fitness Evaluations) e gerações para encontrar uma solução interessante, reduzir o tempo de execução, e ajudar a evitar a convergência prematura. Nos dois cenários testados, a EE proposta conseguiu encontrar soluções que atendem às restrições, com qualquer uma das possibilidades de métodos, embora haja diferenças de desempenho. Os resultados evidenciam o aumento da complexidade do problema com o aumento da região considerada, além de pontos que podem ser melhorados na abordagem para uma aplicação em tempo real.

Este trabalho está assim organizado. A Seção 2 apresenta trabalhos relacionados. A Seção 3 descreve os conceitos envolvidos no problema e sua modelagem. A Seção 4 descreve a Estratégia Evolutiva adotada e decisões de implementação. A Seção 5 descreve os cenários de simulação usados nos experimentos, cujos resultados são apresentados na Seção 6. A Seção 7 conclui o trabalho e aponta possíveis direções futuras. 


\section{Trabalhos Relacionados}

Os trabalhos [Yang 2010] e [Yang and Benekohal 2011] tratam da otimização de semáforos em um cenário com uma interseção. A função objetivo escolhida é a soma dos atrasos médios de cada tipo de usuário das vias urbanas (veículos ou pedestres), variandose o peso do tempo dos pedestres (chamado de $K$ ) entre 0 e 3, com valores inteiros. As temporizações dos semáforos são dadas como valores inteiros e são atribuídas individualmente para cada faixa de cada rua. Além disso, a otimização decide se uma fase exclusiva de pedestres será adicionada ou não. Os autores usam um algoritmo genético para otimizar as temporizações no cenário escolhido. Visando verificar quais fatores tornavam interessante a adoção de uma fase exclusiva de pedestres, diversas configurações foram avaliadas, variando-se $K$, o tamanho das filas de veículos ao início das simulações, a quantidade de faixas em cada sentido das ruas envolvidas, além da densidade de tráfego. Concluiu-se que, ao aumentar $K$, tende a ser mais interessante não utilizar fases exclusivas de pedestres (abordagem TWC), dado que os pedestres passam a ter mais importância na função objetivo. Observou-se que usar a EPP é mais apropriada para fluxos intensos de pedestres e veículos que são perpendiculares. As principais limitações do trabalho são: o uso de variáveis inteiras para a temporização, afetando a precisão da otimização; não ter avaliado outras meta-heurísticas ou melhorias; e a otimização para apenas uma interseção.

Os trabalhos [Gao et al. 2019] e [Gao et al. 2017] realizam uma otimização visando a minimização dos atrasos totais dos veículos e dos pedestres. Os autores elaboraram um modelo matemático para o movimento dos veículos e pedestres. O modelo é macroscópico e depende de algumas considerações: as proporções de veículos e pedestres que se movimentam em cada direção são conhecidas em todas as ruas do cenário; e não são consideradas fases exclusivas de pedestres. O modelo considera a movimentação dentro de intervalos (ou blocos) de tempo de duração arbitrária (não informada pelos autores). Com esta modelagem, a proposta fornece a alocação de fases pré-definidas para blocos de tempo no futuro, permitindo reordenar as permissões de tráfego com base nas demandas. Cada fase ocupa ao menos um bloco inteiro. São testados dois tipos de meta-heurísticas, associados ou não com um algoritmo de busca local. Para resolver o problema para os dois objetivos, os autores usam o conceito da dominância de Pareto [Eiben and Smith 2015] para encontrar as melhores soluções, em que melhorar o valor de um objetivo pede a piora no outro objetivo, um método comum e relevante na otimização multi-objetivo. Contudo, o uso de slots de tempo e de uma abordagem macroscópica limita a precisão da abordagem destes trabalhos.

O trabalho [Ishaque and Noland 2005], embora não envolva a otimização, traz observações importantes. Os autores conduziram simulações de tráfego microscópicas, que envolviam diversos tipos de veículos, além da presença de pedestres. O cenário usado envolve quatro interseções. Foram variados o fluxo de veículos e a duração dos ciclos de operação dos semáforos, mantendo-se constantes os tempos das fases que envolvem pedestres. Também foi considerada a adoção de uma ou duas fases exclusivas de pedestres. Os atrasos para cada tipo de veículo e para os pedestres são analisados para descobrir medidas que podem ser feitas para diminuí-los. Os autores concluem que aumentar a duração do ciclo aumenta a passagem de veículos, até o valor de $90 \mathrm{~s}$. Por outro lado, a duração de $72 \mathrm{~s}$ minimiza o atraso para os carros de passeio. Os pedestres, por outro lado, têm seus atrasos minimizados com ciclos de $45 \mathrm{~s}$, o que indica um conflito entre os interesses de veículos e pedestres. Os autores também observam que é importante ponderar os atrasos 
dos diferentes meios de transporte para atender às diferentes demandas, no caso de uma otimização mono-objetivo. Usar um peso de 2 para os pedestres e dar mais importância a veículos que transportam passageiros (ônibus e táxis) consegue deslocar a solução ótima para ter uma duração de ciclo entre 60 e $72 \mathrm{~s}$, representando melhor compromisso entre os interesses do cenário. No entanto, o estudo simplifica o comportamento dos pedestres, considerando que eles sempre seguem as regras de trânsito e que não interagem fisicamente, não limitando o número de pedestres que atravessam simultaneamente.

O trabalho [Vallyon et al. 2008] avalia a interação de pedestres com os semáforos sob dois aspectos: pela observação dos seus comportamentos em um cenário real na Nova Zelândia, envolvendo a realização de questionários por alguns dos pedestres, e uma avaliação via simulações microscópicas e otimização. Pela observação dos pedestres e pelo questionário, concluiu-se que, quanto mais populosa uma cidade é e quanto mais congestionamentos de trânsito ela tem, maior é o atraso sofrido pelos pedestres nos seus trajetos e maiores são suas percepções de tempo de espera nos cruzamentos. Além disso, maior é a propensão deles a atravessar durante sinais vermelhos ou vermelhos intermitentes, violando as normas de trânsito e aumentando o risco de acidentes. A avaliação via simulação e otimização considera seis cenários, com quantidades variáveis de interseções. Foi observado que realizar a otimização considerando o atraso por pessoa - assumindo que um carro de passageiros transporta, em média, 1,4 pessoa por veículo - causa melhorias significativas no atraso dos pedestres, entre $26 \%$ e $45 \%$, em relação à temporização padrão. O atraso dos veículos, com esta otimização, aumentou levemente em alguns cenários, mas foi reduzido em outros. Outras medidas, como fundir fases que permitem movimentos similares de veículos ou aumentar a duração dos ciclos, provocaram melhorias adicionais para a maioria dos cenários.

\section{Modelagem do Problema}

O problema considerado é a otimização das temporizações de semáforos em interseções para agilizar a passagem de veículos e pedestres. O funcionamento dos sinais de trânsito é regido pelas fases que os compõem, os movimentos que elas permitem e suas durações. Assim, o objetivo é, dadas filas de veículos e pedestres em ruas de um ou mais cruzamentos, fazer com que tais indivíduos saiam o mais rápido possível da região. Para simplificar o problema, as ordens de abertura dos semáforos são imutáveis, excluindo o componente combinatório da otimização, e nenhum outro veículo ou pedestre entra no cenário durante o experimento. A função objetivo é dada pelos atrasos sofridos pelos indivíduos. Fatores também relevantes são a quantidade de FES e o tempo de execução, para que seja verificada a viabilidade de aplicar a otimização em um sistema em tempo real. Os dois cenários avaliados assumem o uso de uma fase exclusiva de pedestres (abordagem EPP) e fases que combinam pedestres e veículos, sem exclusividade (abordagem TWC). Utilizando informações da literatura, foi elaborada neste trabalho a seguinte modelagem para o problema, considerando a TWC em uma interseção:

$$
\begin{aligned}
\operatorname{minimizar} & f\left(T_{\text {verde }, 1}, T_{\text {verde }, 2}, \ldots, T_{\text {verde, },}\right)=D_{\text {med,car }}+2 D_{\text {med,ped }}, i \in\left[1, N_{d}\right] \\
\text { s.a. } & Q_{\text {car }, i}, Q_{\text {ped }, i}, Q_{x} \in \mathbf{Z}^{+}, i \in\left[1, N_{d}\right] \text { (filas - condições de tráfego) } \\
0 & \leq D_{\text {max }, \text { ped }} \leq 60 \mathrm{~s} \\
0 & <\sum_{i=1}^{N_{d}}\left(T_{\text {verde }, i}+T_{\text {amarelo }, i}+T_{\text {fimpedestres }, i}\right) \leq 90 \mathrm{~s}
\end{aligned}
$$




$$
\begin{aligned}
& 3,2+\frac{l_{\text {faixa }}}{V_{\text {ped }}}+0,27 \max _{i=1}^{N_{d}}\left(Q_{p, i}\right) \leq T_{\text {verde }, i} \\
& 1 \leq T_{\text {verde }, i} \\
& D_{x}, T_{x} \in \mathbf{R}^{+}
\end{aligned}
$$

onde $D_{\text {med,car }}=\sum_{i=1}^{N_{\text {veh }}} \frac{D_{\text {car }, i}}{N_{\text {veh }}}$ e $D_{\text {med,ped }}=\sum_{i=1}^{N_{\text {ped }}} \frac{D_{\text {ped }, i}}{N_{\text {ped }}}$ correspondem aos atrasos médios de deslocamento dos veículos e dos pedestres, extraídos da simulação de tráfego; $N_{v e h}$ e $N_{p e d}$ são o número de veículos e pedestres envolvidos na simulação de tráfego; $T_{\text {verde, } i}$ é o tempo em verde do semáforo $i ; N_{d}$ é o número de direções existentes no cruzamento, ou seja, a quantidade de ruas que possuem tráfego direcionado a ele; $T_{\text {amarelo }, i}$ é o tempo em amarelo do semáforo $i$, sendo todos assumidos como $3 \mathrm{~s}-$ valor recomendado pela plataforma de simulação e frequentemente usado por trabalhos relacionados [Yang 2010, Yang and Benekohal 2011, Ishaque and Noland 2005], dado que afeta a segurança no trânsito; $T_{\text {fimpedestres }, i}$ é o tempo durante o qual o sinal de alerta para pedestres (vermelho intermitente) fica ativo, sendo outro intervalo de segurança para limpar as vias urbanas, assumido como $5 \mathrm{~s}$, dado pela própria plataforma de simulação em função das dimensões das interseções; $D_{\text {max,ped }}=\max _{i=1}^{N_{\text {ped }}} D_{\text {ped }, i}$ corresponde ao maior atraso sofrido por um pedestre envolvido na simulação; $Q_{c a r, i}, Q_{p e d, i}$ são as filas de veículos e pedestres na direção $i$, que são condições da simulação de tráfego em questão; $l_{\text {faixa }}$ é o comprimento da faixa de pedestres; e $V_{\text {ped }}$ corresponde à velocidade de um pedestre caminhando, assumida como 1,2 m/s [Yang 2010].

A função objetivo (Equação 1) é uma combinação linear dos atrasos médios dos veículos e dos pedestres. Atribui-se peso 2 para o atraso dos pedestres, considerado um bom equilíbrio entre pedestres e veículos [Yang 2010, Yang and Benekohal 2011, Ishaque and Noland 2005]. A Equação 3 é o limite máximo dado ao atraso máximo dos pedestres, formulada a partir de estudos na direção da elaboração de um manual de segurança no trânsito para a Alemanha [Brilon 1994]. O valor de $60 \mathrm{~s}$ se justifica por informações da literatura. Segundo [Ishaque and Noland 2005], pedestres começam a ficar impacientes a partir de 30 segundos de espera. Além disso, de acordo com observações empíricas [Vallyon et al. 2008, Virkler 1998], quanto mais tempo os pedestres esperam, mais eles se arriscam para atravessar as ruas e economizar tempo, o que se relaciona à observação anterior. A Equação 4 é relativa a duração total de um ciclo. Ciclos acima de $90 \mathrm{~s}$ devem ser evitados, uma conclusão derivada da restrição sobre o atraso máximo dos pedestres [Brilon 1994]. A Equação 5 é a duração mínima que uma fase que permita a passagem de pedestres deve ter, extraída de [Yang 2010] e originária do Highway Capacity Manual ${ }^{1}$, documento de referência usado por engenheiros de tráfego dos Estados Unidos. A Equação 6 é um valor arbitrário para o tempo mínimo em verde de qualquer semáforo, para evitar simulações em que o tempo em verde seria 0 para uma direção com veículos, nunca encerrando. As Equações 4, 5 e 6 dizem respeito às variáveis de decisão do problema. No entanto, a Equação 3 é relativa a um resultado da simulação - o atraso máximo dos pedestres. Para o uso de uma fase exclusiva de pedestres, a modelagem anterior foi adaptada para a forma a seguir:

$$
\begin{gathered}
\operatorname{minimizar} f\left(T_{\text {verde }, 1}, T_{\text {verde }, 2}, \ldots, T_{\text {verde }, i}, T_{\text {ped }}\right)=D_{\text {med,car }}+2 D_{\text {med,ped }}, i \in\left[1, N_{d}\right] \\
\text { s.a. } Q_{\text {car }, i}, Q_{\text {ped }, i}, Q_{x} \in \mathbf{Z}^{+}, i \in\left[1, N_{d}\right] \text { (filas - condições de tráfego) }
\end{gathered}
$$

\footnotetext{
${ }^{1}$ Edição mais atual: $h t t p: / / w w w . t r b . o r g / p u b l i c a t i o n s / h c m 6 e . a s p x$.
} 


$$
\begin{aligned}
& 0 \leq D_{\text {max }, \text { ped }} \leq 60 \mathrm{~s} \\
& 0<\sum_{i=1}^{N_{d}}\left(T_{\text {verde }, i}+T_{\text {amarelo }, i}+T_{\text {ped }}+T_{\text {fimpedestres }, i}\right) \leq 90 \mathrm{~s} \\
& 3,2+\frac{l_{\text {faixa }}}{V_{\text {ped }}}+0,27 \max _{i=1}^{N_{d}}\left(Q_{p, i}\right) \leq T_{\text {ped }} \\
& 1 \leq T_{\text {verde, } i} \\
& D_{x}, T_{x} \in \mathbf{R}^{+}
\end{aligned}
$$

Uma variável adicional aparece, $T_{p e d}$, correspondente à duração da fase exclusiva de pedestres. Além disto, $T_{\text {fimpedestres }}$, presente na Equação 11, perde o índice de direção, dado que somente há um período de transição entre uma fase com pedestres e outra com veículos, diferentemente do que ocorre no TWC.

Para múltiplas interseções, cada interseção adicional adiciona às variáveis de decisão tempos em verde relativos às suas direções com semáforos, e novas restrições de duração dos ciclos - 1 por interseção adicional -, dos tempos em verde dos pedestres -1 por fase que contém pedestres na interseção adicional - e dos tempos em verde para qualquer direção - 1 por direção na interseção. Novas interseções também adicionam novas filas em cada nova rua.

\section{Algoritmo Utilizado}

O Algoritmo Genético (AG) é um dos mais frequentemente utilizados neste problema [Yang and Benekohal 2011, Jin et al. 2017, Ma et al. 2015, Sanchez-Medina et al. 2010]. No entanto, a Estratégia Evolutiva (EE) [Beyer and Schwefel 2002] se mostrou mais eficiente em avaliações iniciais nos cenários utilizados aqui, sendo, portanto, escolhida. A EE é um algoritmo evolucionário similar a um AG, possuindo crossover (ou recombinação), mutação e seleção de sobreviventes. A principal diferença é que a EE não emprega um mecanismo de seleção de pais, sendo os filhos gerados a partir de qualquer indivíduo da população atual, permitindo uma maior variabilidade.

$\mathrm{Na}$ EE empregada, indivíduos possuem as variáveis de decisão - temporizações - codificadas como valores reais. A população é inicializada aleatoriamente, com uma distribuição uniforme dentro do espaço de busca de cada variável. É típico das EEs gerar uma quantidade de filhos $(\lambda)$ muito superior à quantidade de pais ( $\mu$ - o tamanho da população em todas as gerações), fornecendo uma busca acelerada e, ao mesmo tempo, boa exploração em regiões promissoras. Uma proporção recomendada é o valor aproximado de 7 [Eiben and Smith 2015]. Empiricamente, observou-se que $\lambda / \mu=8$ foi uma boa configuração para os cenários considerados. Foram usados $\mu=20$ e $\lambda=160$. Também foi usado elitismo, preservando o melhor indivíduo a cada geração. Para gerar filhos a partir da população foi utilizado o crossover BLX- $\alpha$ [Eshelman and Schaffer 1993]. Dados dois pais $p_{1}$ e $p_{2}$, um filho $f$ é gerado da seguinte forma:

$$
f=p_{1}+\beta\left(p_{2}-p_{1}\right),
$$

onde $\beta \in U(-\alpha, 1+\alpha)$, ou seja, é gerado de uma distribuição uniforme. O valor adotado para $\alpha$ é de 0,5 e a probabilidade de recombinação, 0,8. Cada operação gera 1 filho. 
Outra característica da EE que melhora seu desempenho é o uso de mutações com parâmetros auto-adaptativos. Neste trabalho, foi usada uma modificação da mutação creep [Beyer and Schwefel 2002], que a adapta ao comportamento da função objetivo. O mecanismo permite que o algoritmo escolha, durante a sua operação, os parâmetros mais interessantes a cada geração, que dependem da função objetivo. Uma mutação creep gera perturbações normais sobre cada variável de decisão do indivíduo; suas médias foram mantidas como 0 . Os desvios-padrão da mutação de cada dimensão $\left(\sigma_{i}\right.$, onde $i$ corresponde a uma dimensão) são alterados a cada rodada. Para isto, eles são adicionados aos genótipos dos indivíduos e sofrem alterações pelo crossover. Antes de cada fase de mutação, eles são alterados, seguindo a seguinte equação:

$$
\sigma_{i}^{\prime}=\sigma_{i} \times e^{\tau^{\prime} N(0,1)+\tau N_{i}(0,1)},
$$

sendo $\sigma_{i}$ o desvio-padrão da dimensão $i, \sigma_{i}^{\prime}$ seu próximo valor, $\tau^{\prime}$ a taxa de aprendizado global, que ajusta as perturbações de todas as dimensões, $\tau$ a taxa de aprendizado individual, que ajusta as perturbações individuais em cada dimensão, $N(0,1)$ uma perturbação normal que vale para todas as dimensões e $N_{i}(0,1)$ uma perturbação normal individual de cada dimensão. Com os valores calculados de desvio-padrão, as variáveis de cada dimensão do genótipo de um novo indivíduo são calculadas da seguinte forma:

$$
x_{i}^{\prime}=x_{i}+\sigma_{i}^{\prime} \times N_{i}(0,1) .
$$

Os valores adotados para $\tau^{\prime}$ e $\tau$ foram, respectivamente, $\left.1 / \sqrt{(} 2 n\right)$ e $\left.\left.1 / \sqrt{(} 2 \sqrt{(} n\right)\right)$ [Beyer and Schwefel 2002]. Para evitar que os desvios-padrão se aproximem demais de 0, o que faria o algoritmo estagnar, o limite inferior $\epsilon_{0}=10^{-5}$, obtido através de experimentos iniciais, foi utilizado. Um limite superior $\epsilon_{\max }$ também foi adotado: corresponde a $60 \%$ do intervalo de busca de cada variável, para evitar que os passos fiquem muito altos e que as soluções se concentrem nos extremos dos intervalos. Como os passos tendem a diminuir ao longo da execução do algoritmo, escolheu-se utilizar um desvio-padrão inicial alto para todas as variáveis, para que as primeiras gerações realizem uma maior exploração. Por isto, os tamanhos de passo iniciais correspondem a $60 \%$ dos intervalos de busca de cada variável. Por fim, a probabilidade de mutação é $100 \%$, dado o caráter auto-adaptativo da mutação empregada.

Foi avaliada a eficácia de um mecanismo de busca adicional para a EE. O procedimento é a mutação utilizada na Evolução Diferencial (ED) [Storn and Price 1997]. A variação escolhida foi a rand/2/bin. Ela é efetuada após a fase de mutação. Efetivamente, o procedimento escolhe dois outros indivíduos aleatórios da população e soma a diferença entre eles ao indivíduo atual. Esta diferença é ponderada por um peso - aqui adotou-se 0,5. Caso encontre uma solução melhor que a anterior, a solução anterior é substituída. Dois mecanismos de tratamento de restrições foram empregados alternadamente:

1) Penalização Aditiva [Eiben and Smith 2015]: modifica a função objetivo, adicionando um termo de penalização calculado em função das violações das restrições, sendo as violações métricas que indicam quão afastada da região factível uma dada solução está. Supondo um conjunto de variáveis de decisão $\mathbf{x}$, uma função objetivo $f(\mathbf{x})$, uma restrição de desigualdade $g(\mathbf{x}) \leq 0$ e uma restrição de igualdade $h(\mathbf{x})=0$, uma nova função é criada a partir das violações. Foi escolhida a seguinte formulação:

$$
f_{2}(x)=f(x)+k P(\mathbf{x}),
$$




$$
P(\mathbf{x})=\max (0, g(\mathbf{x}))+\max (0,(|h(\mathbf{x})|-\epsilon)),
$$

No caso de mais restrições de igualdade ou desigualdade, termos similares são somados ao valor de $P$.

2) TS-R [Deb 2000]: usa uma seleção por torneio com três regras de comparação par-a-par:

- Entre dois indivíduos factíveis, o de melhor aptidão é selecionado;

- Entre um indivíduo factível e um infactível, o factível é selecionado;

- Entre dois indivíduos infactíveis, o que oferece a menor violação das restrições é selecionado.

Além destes mecanismos, a terceira e a quarta restrições do problema, presentes na Seção 3, referentes aos tempos mínimos em verde de fases com semáforos, representam limites inferiores, sendo incorporadas aos intervalos de busca. Indivíduos que violem estas restrições em alguma variável têm estas variáveis truncadas para o limite em questão.

Dois métodos diferentes de seleção de sobreviventes foram empregados, a depender do tratamento de restrições adotado. Para a penalização aditiva, foi usada uma seleção geracional, selecionando $\mu$ indivíduos para compor a população da próxima geração a partir dos $\lambda$ filhos gerados em uma geração, sendo $\lambda / \mu=8$. Os filhos são ordenados em ordem crescente de aptidão, dado que o problema considerado é de minimização. Para o TS-R, a seleção por torneio proposta pelo método foi utilizada.

Com inspiração na variante do AG chamada AMGA2 [Jin et al. 2017], foi usado um banco de dados de soluções, que contém as variáveis de decisão e todas as métricas relevantes. Ao avaliar uma solução, consulta-se o banco de dados. Se uma solução igual já está armazenada, as métricas são recuperadas de lá, a avaliação é pulada e o contador de FES não é incrementado. Esta é uma medida que traz dois benefícios. O primeiro é economizar simulações, agilizando a busca. O segundo é analisar o desempenho do algoritmo: muitos resgates do banco de dados indicam a produção de muitas soluções repetidas, sinal de convergência prematura. Para reduzir ainda mais o custo computacional das simulações, elas foram paralelizadas. Na criação da população inicial e após as mutações de cada geração, todos os indivíduos da população ou do conjunto de filhos são avaliados em paralelo.

Neste trabalho, realizou-se uma implementação própria da EE, integrada com o SUMO. O ambiente de simulação foi uma máquina com processador Intel Xeon com 4 núcleos físicos e 4 lógicos de clock máximo de $2,91 \mathrm{GHz}$ em paralelo ou $3,46 \mathrm{GHz}$ em serial, e 8 GB de RAM. As simulações do SUMO possuem diversos componentes aleatórios. Para torná-las determinísticas e reprodutíveis, uma semente foi fixada para o gerador de números aleatórios do simulador, de valor 23423, um valor padrão ${ }^{2}$. As simulações se encerram assim que todos os veículos e pedestres cumprem seus trajetos.

\section{Cenários de Simulação}

A abordagem foi testada em dois cenários de simulação. O Cenário 1 possui apenas uma interseção, sendo mais simples, porém útil para testar a abordagem e aprender características do problema. Sua topologia foi extraída da plataforma OpenStreetMap ${ }^{3}$, a

\footnotetext{
${ }^{2}$ Mais informações em https://sumo.dlr.de/docs/Simulation/Randomness.html.

${ }^{3}$ https://www.openstreetmap.org.
} 
partir da cidade do Rio de Janeiro e é ilustrada pela Figura 1a. Ele possui um cruzamento entre quatro ruas de mão única. A rua com tráfego de oeste para leste possui mais faixas e mais veículos. A rua da direção sul possui veículos que desejam trafegar na direção leste e uma fila de pedestres que desejam atravessar o cruzamento e seguir na direção norte. Há 24 veículos que partem da direção oeste, 12 veículos que partem da direção sul e 32 pedestres. Estas quantidades são arbitrárias. Os dois semáforos da rua compõem a seguinte sequência de fases:

- Verde para a rua Sul (veículos e pedestres) - ajustável;

- Verde para os veículos da rua Sul e vermelho para os pedestres - 5 segundos;

- Amarelo para os veículos da rua Sul e vermelho para os pedestres - 3 segundos;

- Verde para os veículos da rua Oeste - ajustável;

- Amarelo para os veículos da rua Oeste - 3 segundos.

Neste cenário, não há fases exclusivas de pedestres. Há 2 variáveis de decisão tempos em verde de 2 fases. Também há 1 restrição sobre a duração do ciclo de operação da interseção do cenário, 2 restrições de limite mínimo para o tempo em verde e 1 restrição sobre o atraso máximo dos pedestres, totalizando 4.

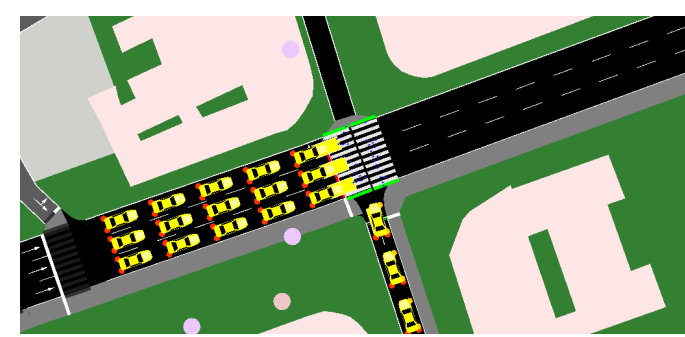

(a) Cenário 1: uma interseção.

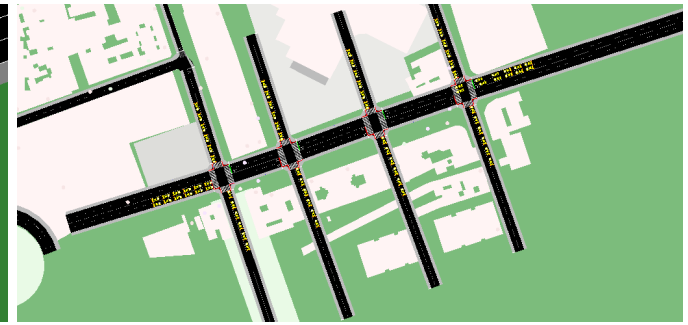

(b) Cenário 2: quatro interseções.

Figura 1. Cenários de simulação.

O Cenário 2 é maior, comparável a cenários de trabalhos relacionados, tendo 4 interseções. Cada uma das 4 ruas que compõem cada interseção tem tráfego em mão dupla e calçadas para pedestres. As ruas no sentido horizontal possuem duas faixas para veículos em cada sentido, enquanto as verticais possuem apenas uma. Todas as faixas de veículos permitem movimentos em todas as direções. Cada uma das quatro interseções possui quatro semáforos, que compõem a mesma sequência de fases:

- Verde para veículos vindos da direção leste - ajustável;

- Amarelo para veículos vindos da direção leste - 3 segundos;

- Verde para veículos vindos da direção sul - ajustável;

- Amarelo para veículos vindos da direção sul - 3 segundos;

- Verde para veículos vindos da direção oeste - ajustável;

- Amarelo para veículos vindos da direção oeste - 3 segundos;

- Verde para veículos vindos da direção norte - ajustável;

- Amarelo para veículos vindos da direção norte - 3 segundos;

- Fase exclusiva de pedestres - ajustável;

- Fase com todos os semáforos em vermelho - 5 segundos.

O Cenário 2 possui fases exclusivas de pedestres, o que aumenta o número de variáveis do problema - em 1 por cruzamento, sem perder a relação com cenários reais. 
Cada interseção possui 5 temporizações ajustáveis. Sendo quatro interseções, há um total de 20 variáveis reais a se ajustar, além de 29 restrições; 1 de atraso máximo dos pedestres, 4 relativas às durações dos ciclos, 20 de tempo mínimo em verde para veículos, e 4 de tempo mínimo em verde para as fases exclusivas de pedestres. Vinte e quatro veículos partem das ruas do sentido horizontal e 48 saem do sentido vertical, totalizando 72; seus movimentos são distribuídos uniformemente entre todas as direções. Cada uma das ruas do sentido vertical possui entre 4 e 6 pedestres, totalizando 42. Aproximadamente 50\% deles atravessam 1 cruzamento; a outra metade atravessa 2. Seus movimentos também são distribuídos igualmente entre as possíveis direções.

\section{Resultados}

Para o Cenário 1, foram testadas quatro variações da EE: EE1 (penalização aditiva, com $K=2$ ); EE2 (penalização aditiva, com $K=2$ e busca adicional); EE3 (uso do TS-R); e EE4 (uso do TS-R e busca adicional). Cada variação foi executada por 5 rodadas. O critério de parada foram 5000 FES. A Tabela 1 apresenta as medidas para a melhor solução de cada algoritmo, com valores decimais arredondados para três casas. Este arredondamento será feito para quaisquer outras tabelas do relatório. $T_{\text {verde, } 1}$ corresponde ao tempo em verde da rua da direção Sul (com veículos e pedestres), $T_{\text {verde }, 2}$ corresponde ao tempo em verde da rua da direção Oeste (apenas veículos, mas em maior quantidade), $D_{\text {med,car }}$ representa o atraso médio dos veículos, $D_{\text {med,ped }}$ é o atraso médio dos pedestres, $D_{\text {max,ped }}$ é o atraso máximo dos pedestres e $C$ é a duração do ciclo da operação. Pode-se observar que a EE1, apenas com a penalização aditiva, foi o único algoritmo a achar o mínimo de aptidão de 25,593. Ela obteve uma aptidão melhor que a EE2, com busca adicional. A busca adicional ofereceu uma melhoria em combinação com o TS-R, porém, dado que nem a EE3 nem a EE4 atingiram a mesma aptidão que a EE1, este benefício parece não ser significativo. Pelo mesmo motivo, o TS-R aparenta não ser a melhor estratégia para buscar soluções de melhor qualidade, ao menos para este cenário. Apesar das diferenças entre os resultados, as quatro abordagens obtiveram soluções que têm valores reduzidos de atraso médio e estando afastados dos limites superiores de atraso máximo de pedestres e duração do ciclo. Vale mencionar que nenhuma solução foi recuperada do banco de dados, indicando que a EE gerou soluções variadas.

Tabela 1. Cenário 1 - Resultados para as melhores soluções (em segundos).

\begin{tabular}{|c|c|c|c|c|c|c|c|}
\hline Algoritmo & $T_{\text {verde }, 1}$ & $T_{\text {verde }, 2}$ & Aptidão & $D_{\text {med,car }}$ & $D_{\text {med,ped }}$ & $D_{\text {max,ped }}$ & $C$ \\
\hline EE1 & 25,593 & 4,000 & 64,91 & 22,75 & 21,08 & 29,759 & 46,593 \\
\hline EE2 & 25,593 & 5,000 & 65,77 & 21,93 & 21,92 & 30,6 & 47,593 \\
\hline EE3 & 25,593 & 4,097 & 66.6 & 22,759 & 21,92 & 30,6 & 46,690 \\
\hline EE4 & 27,123 & 5,000 & 65.9 & 22,06 & 21,92 & 30,6 & 49,123 \\
\hline
\end{tabular}

Tabela 2. Cenário 1 - Estatísticas restantes da aptidão.

\begin{tabular}{|c|c|c|c|c|c|c|}
\hline Algoritmo & Pior & Média & Mediana & $\begin{array}{c}\text { Desvio } \\
\text { Padrão }\end{array}$ & Factibilidade & $\begin{array}{c}\text { Tempo de } \\
\text { Execução Médio (s) }\end{array}$ \\
\hline EE1 & 66,6 & 65,752 & 65,74 & 0,845 & $100 \%$ & 702,842 \\
\hline EE2 & 66,6 & 66,434 & 66,6 & 0,371 & $100 \%$ & 672,238 \\
\hline EE3 & 66,83 & 66,658 & 66,6 & 0,100 & $100 \%$ & 719,946 \\
\hline EE4 & 66,66 & 66,472 & 66,6 & 0,32 & $100 \%$ & 733,538 \\
\hline
\end{tabular}


A Tabela 2 apresenta estatísticas dos valores de aptidão, a taxa de factibilidade e o tempo médio de execução. Ela indica novamente que a EE1 foi a melhor opção para este cenário: encontrou a melhor solução, as menores aptidões média e mediana e o segundo menor tempo de execução.

No Cenário 2, apenas duas variantes da EE são consideradas: a EE com a penalização aditiva e a com o TS-R [Deb 2000]. Testes iniciais indicaram que $K=3$ é um valor mais eficaz que $K=2$ para a penalização aditiva aqui, sendo adotado. Os critérios de parada adotados foram 8000 FES e 900 gerações. Cada variante foi executada por 5 rodadas, dados os tempos de execução mais altos das otimizações neste cenário e dificuldades encontradas com a ferramenta de simulação. A Tabela 3 mostra a melhor solução, encontrada pelas duas variações. Cada linha indica a temporização de um semáforo, da direita para a esquerda, e cada variável $T_{\text {verde }, i}$ lida da esquerda para a direita diz respeito à fase que permite a passagem de veículos vindos de uma direção, no sentido horário, começando da direção leste. Estas direções têm como referência a Figura 1b. A Tabela 4 indica os valores das métricas extraídas da simulação a partir das melhores soluções, além do tempo médio de execução. Já a Tabela 5 indica estatísticas sobre o valor da aptidão e a taxa de factibilidade. Avaliando as Tabelas 3, 4 e 5, pode se observar que os dois métodos conseguiram encontrar a mesma solução e tiveram uma taxa de factibilidade de $100 \%$. No entanto, a penalização aditiva forneceu mais soluções de melhor qualidade, dados os valores médio e pior da aptidão, a mediana e o desvio-padrão.

Os resultados obtidos são coerentes com a literatura. O uso da fase exclusiva de pedestres como a última fase do ciclo impõe um peso mais forte aos pedestres. Por isto, sua duração é maior que a de qualquer outra fase e a proporção entre os atrasos médios dos pedestres e dos veículos é diferente do cenário anterior (Tabela 1). Enquanto, no Cenário 1, os atrasos tinham valores muito próximos, com uma diferença máxima de 1,67 s, há uma discrepância muito maior no Cenário 2, de 98,94 s. Deve-se observar, no entanto, que, no Cenário 2, alguns veículos e alguns pedestres precisam passar por mais de um cruzamento para cumprir seus trajetos, o que não ocorre no Cenário 1.

As Figuras 2 e 3 mostram as evoluções das proporções de soluções factíveis nas populações, respectivamente em função das FES e das gerações. Elas indicam que o TSR não conseguiu estabilizar a população em uma proporção alta de soluções factíveis, permanecendo com ela abaixo de $60 \%$ em todas as rodadas, com muitas oscilações entre as gerações. O método TS-R tem a capacidade de encontrar com menos FES uma solução factível, porque ele não avalia soluções infactíveis, diferentemente da penalização aditiva. Porém, pede mais gerações para achar uma solução factível, o que explica o maior tempo de execução, visto que apenas as FES são paralelizadas. A penalização aditiva é o único método que, em algum momento da execução, alcança 100\% de soluções factíveis dentro da população, além de conseguir manter a proporção de soluções factíveis mais estável que o TS-R, mostrando fazer uma busca mais eficiente.

Por fim, vale mencionar que nenhuma solução foi recuperada do banco de dados neste cenário. Ao se usar outras técnicas de crossover para EEs - intermediário ou discreto - em avaliações iniciais, estas consultas ocorreram. Ou seja, o BLX- $\alpha$ consegue diversificar mais as soluções, de modo que elas não se repitam. 
Tabela 3. Cenário 2 - Melhor solução encontrada (temporizações em segundos).

\begin{tabular}{|c|c|c|c|c|c|c|}
\hline Semáforo & $T_{\text {verde }, 1}$ & $T_{\text {verde }, 2}$ & $T_{\text {verde }, 3}$ & $T_{\text {verde }, 4}$ & $T_{\text {ped }}$ & $C$ \\
\hline 1 & 4,587 & 5,935 & 2,703 & 11,491 & 30,280 & 71,995 \\
\hline 2 & 12,109 & 1,708 & 17,216 & 7,621 & 27,812 & 83,466 \\
\hline 3 & 1 & 7,612 & 1 & 9,720 & 23,221 & 59,553 \\
\hline 4 & 3,280 & 4,007 & 6,829 & 4,260 & 18,573 & 53,950 \\
\hline
\end{tabular}

Tabela 4. Cenário 2 - Resultados para as melhores soluções (em segundos).

\begin{tabular}{|c|c|c|c|c|c|}
\hline Algoritmo & Aptidão & $D_{\text {med,car }}$ & $D_{\text {med,ped }}$ & $D_{\text {max,ped }}$ & $\begin{array}{c}\text { Tempo de Execução } \\
\text { Médio (s) }\end{array}$ \\
\hline EE + Aditiva & 166,53 & 121,47 & 22,53 & 59,76 & 2886,971 \\
\hline EE + TS-R & 166,53 & 121,47 & 22,53 & 59,76 & 3322,045 \\
\hline
\end{tabular}

Tabela 5. Cenário 2 - Estatísticas restantes.

\begin{tabular}{|c|c|c|c|c|c|}
\hline Algoritmo & Pior & Média & Mediana & Desvio-padrão & Taxa de Factibilidade \\
\hline EE + Aditiva & 176,5 & 169,304 & 168,24 & 4,143 & $100 \%$ \\
\hline EE + TS-R & 191,09 & 182,6 & 184,72 & 9,365 & $100 \%$ \\
\hline
\end{tabular}

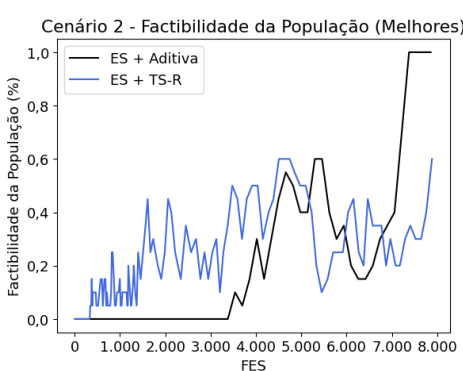

(a) Melhores rodadas.

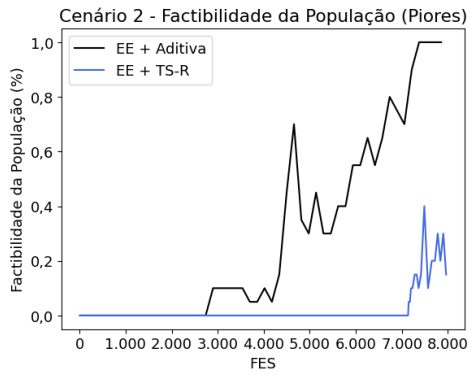

(b) Piores rodadas.

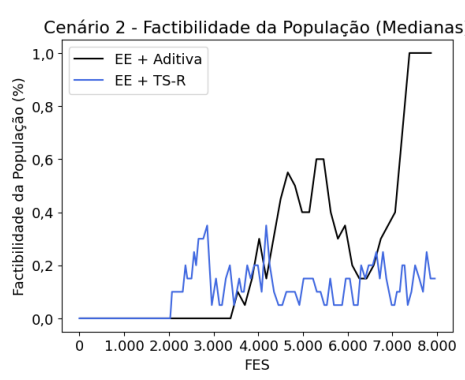

(c) Rodadas (aptidão mediana).

Figura 2. Cenário de Simulação 2 - proporções de soluções factíveis nas populações por FES.

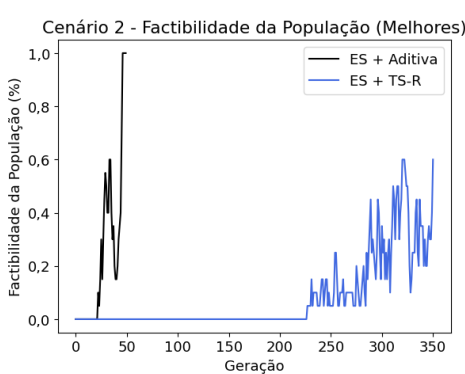

(a) Melhores rodadas.

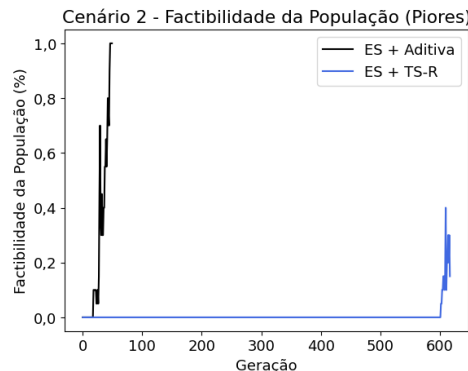

(b) Piores rodadas.

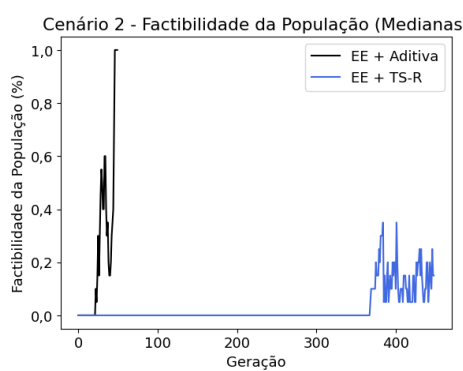

(c) Rodadas (aptidão mediana).

Figura 3. Cenário de Simulação 2 - proporções de soluções factíveis nas populações por gerações.

\section{Conclusão}

Este trabalho avaliou a aplicação de variações de uma Estratégia Evolutiva (EE) para a otimização da temporização de sinais de trânsito em cenários urbanos, com o uso de simulações microscópicas. As variantes usaram duas técnicas de tratamento de restrições e diversas modificações. Foram testados o TS-R e a Penalização Aditiva combinada com 
uma seleção de sobreviventes geracional como tratamentos de restrições, além de outros componentes para acelerar a busca e reduzir o custo computacional das simulações.

Os resultados mostram o rápido aumento da complexidade do problema com a quantidade de semáforos. Entre o Cenário 1 e o Cenário 2, nota-se uma diferença expressiva na dificuldade em se alcançar a região factível e em manter a população nela. No Cenário 2, o algoritmo mais rápido usou a Penalização Aditiva, encontrando uma solução factível em aproximadamente 16 min. (2740 FES em 17 gerações). Já no Cenário 1 , uma solução factível é encontrada dentro de $60 \mathrm{~s}$. Desta forma, o Cenário 1 permitiria a operação em tempo real, ao passo que, no Cenário 2, uma otimização poderia ser executada a cada 11 ciclos, respondendo mais lentamente às condições de tráfego. Caso as condições não se alterem com muita frequência, esta abordagem poderia ser aplicada para encontrar uma temporização que siga as restrições usadas na modelagem. No entanto, cenários mais dinâmicos pedem aprimoramentos. Tais melhorias precisam contornar as limitações da abordagem. Por exemplo, outros mecanismos de busca em combinação com o algoritmo podem melhorar seu desempenho. Além disto, paralelizar outras etapas do algoritmo, como a etapa de mutação, pode ajudar a economizar tempo de processamento. Por fim, escolher um outro método de tratamento de restrições pode ser uma alternativa interessante. Tal método deve ter sinergia com uma EE e ter características benéficas dos dois tratamentos de restrições usados aqui, acelerando a busca e economizando as avaliações de soluções infactíveis.

Por fim, foi possível perceber o impacto da adoção de uma fase exclusiva de pedestres, que induz a otimização a encontrar soluções que privilegiem mais estes usuários, tornando os atrasos sofridos pelos veículos nos seus deslocamentos quase seis vezes maiores que os deslocamentos que os pedestres tiveram nas simulações realizadas.

\section{Agradecimentos}

Este trabalho foi parcialmente financiado pelas agências de fomento CAPES - Código 001, CNPq, FAPERJ, e pelo processo no 15/24494-8, da Fundação de Amparo à Pesquisa do Estado de São Paulo (FAPESP).

\section{Referências}

Beyer, H.-G. and Schwefel, H.-P. (2002). Evolution strategies - A comprehensive introduction. Natural Computing, 1(1):3-52.

Brilon, W. (1994). Traffic engineering and the new German highway capacity manual. Transportation Research Part A: Policy and Practice, 28(6):469-481.

Deb, K. (2000). An efficient constraint handling method for genetic algorithms. Comput. Methods Appl. Mech. Engrg., page 28.

Eiben, A. and Smith, J. (2015). Introduction to Evolutionary Computing. Natural Computing Series. Springer Berlin Heidelberg, Berlin, Heidelberg.

Eshelman, L. J. and Schaffer, J. D. (1993). Real-coded genetic algorithms and intervalschemata. In WHITLEY, L. D., editor, Foundations of Genetic Algorithms, volume 2 of Foundations of Genetic Algorithms, pages 187 - 202. Elsevier. 
Gao, K., Zhang, Y., Zhang, Y., and Su, R. (2017). A meta-heuristic with ensemble of local search operators for urban traffic light optimization. In 2017 IEEE Symposium Series on Computational Intelligence (SSCI), pages 1-8.

Gao, K., Zhang, Y., Zhang, Y., Su, R., and Suganthan, P. N. (2019). Meta-heuristics for bi-objective urban traffic light scheduling problems. IEEE Transactions on Intelligent Transportation Systems, 20(7):2618-2629.

Ishaque, M. M. and Noland, R. B. (2005). Multimodal Microsimulation of Vehicle and Pedestrian Signal Timings. Transportation Research Record, page 8.

Jin, J., Ma, X., and Kosonen, I. (2017). A stochastic optimization framework for road traffic controls based on evolutionary algorithms and traffic simulation. Advances in Engineering Software, 114:348-360.

Lopez, P. A., Behrisch, M., Bieker-Walz, L., Erdmann, J., Flötteröd, Y.-P., Hilbrich, R., Lücken, L., Rummel, J., Wagner, P., and Wießner, E. (2018). Microscopic Traffic Simulation using SUMO. In The 21st IEEE International Conference on Intelligent Transportation Systems. IEEE.

Ma, W., Liao, D., Liu, Y., and Lo, H. K. (2015). Optimization of pedestrian phase patterns and signal timings for isolated intersection. Transportation Research Part C: Emerging Technologies, 58:502-514.

Reed, T. and Kidd, J. (2019). INRIX 2018 Global Traffic Scorecard. Technical report, INRIX.

Sanchez-Medina, J., Galan-Moreno, M., and Rubio-Royo, E. (2010). Traffic Signal Optimization in "La Almozara" District in Saragossa Under Congestion Conditions, Using Genetic Algorithms, Traffic Microsimulation, and Cluster Computing. IEEE Transactions on Intelligent Transportation Systems, 11(1):132-141.

Schmöcker, J.-D., Ahuja, S., and Bell, M. G. (2008). Multi-objective signal control of urban junctions - Framework and a London case study. Transportation Research Part C: Emerging Technologies, 16(4):454-470.

Storn, R. and Price, K. (1997). Differential Evolution - A Simple and Efficient Heuristic for Global Optimization over Continuous Spaces. Journal of Global Optimization, page 19.

Vallyon, C., Binder, S., and Hodgson, S. (2008). Reducing Pedestrian Delays at Traffic Signals. In IPENZ Transportation Group Conference New Plymouth, page 15, New Plymouth, New Zealand.

Virkler, M. R. (1998). Pedestrian Compliance Effects on Signal Delay. Transportation Research Record: Journal of the Transportation Research Board, 1636(1):88-91.

Yang, Z. (2010). Signal Timing Optimization Based on Minimizing Vehicle and Pedestrian Delay by Genetic Algorithm. Dissertação de Mestrado, 2010, University of Illinois at Urbana-Champaign.

Yang, Z. and Benekohal, R. F. (2011). Use of Genetic Algorithm for Phase Optimization at Intersections with Minimization of Vehicle and Pedestrian Delays. Transportation Research Record: Journal of the Transportation Research Board, 2264(1):54-64. 